Indo Global Journal of Pharmaceutical Sciences, 2015; 5(3): 225-232

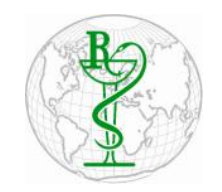

INDO GLOBAL JOURNAL OF

PHARMACEUTICAL SCIENCES

ISSN 2249- 1023

\title{
Development \& Validation of Absorbance Ratio Method for Simultaneous Estimation of Lornoxicam \& Eperisone in their Synthetic Mixture
}

\author{
Jawed Akhtar ${ }^{1 *}$, Jatin Prajapati ${ }^{2}$, Gamal Osman Elhassan ${ }^{3}$, Mohammad Mujahid ${ }^{1}$ \\ ${ }^{1}$ Translam Institute of Pharmaceutical Education \& Research, Meerut, Uttar Pradesh-250001 \\ ${ }^{2}$ Astra Life Care Pvt. Ltd., Ahmedabad \\ ${ }^{3}$ Unaizah College of Pharmacy, Qassim University, KSA
}

\begin{abstract}
Address for Correspondance Jawed Akhtar, jawed.pharmacy @gmail.com

ABSTRACT: The present manuscript describe simple, sensitive, rapid, accurate, precise and economical Q-absorbance ratio method for the simultaneous determination of Lornoxicam (LXM) and Eperisone (EPE) in synthetic mixture. Absorbance ratio method uses the ratio of absorbances at two selected wavelengths, one which is an isoabsorptive point and other being the $\lambda$-max of one of the two components. LXM and EPE show an isoabsorptive point at $291.5 \mathrm{~nm}$ in $0.1 \mathrm{~N}$ methanolic $\mathrm{NaOH}$. The second wavelength used is $254.5 \mathrm{~nm}$, which is the $\lambda$-max of EPE in methanol. The linearity was obtained in the concentration range of $2-16 \mu \mathrm{g} / \mathrm{ml}$ for both LXM and EPE. The concentrations of the drugs were determined by using ratio of absorbances at isoabsorptive point and at the $\lambda$-max of EPE. The method was successfully applied to synthetic mixture because no interference from the tablet excipients was found. The results of analysis have been validated statistically and by recovery studies.(C) 2015 iGlobal Research and Publishing Foundation. All rights reserved.

Eperisone;

Absorption Ratio
\end{abstract}

Method; Synthetic Mixture; Validation.

\section{INTRODUCTION}

LXM is chemically (3E)-6-chloro-3-[hydroxy(pyridin-2ylamino)methylene]-2 methyl-2,3-dihydro-4H-thieno[2,3e][1,2] thiazin-4-one 1,1-dioxide. LXM is an NSAID of the oxicam class with analgesic, anti-inflammatory and antipyretic properties. It inhibits prostaglandin synthesis by inhibiting both cyclooxygenase enzyme (COX-1 and COX-2). EPE is chemically (2RS)-1-(4-ethylphenyl)-2methyl-3- (1-piperidyl) propane-1-one. EPE is an antispasmodic drug. It acts by relaxing both skeletal muscles and vascular smooth muscles, and demonstrates a variety of effects such as reduction of myotonia, improvement of circulation, and suppression of the pain reflex. The review of literature revealed that various involving spectrophotometry have been reported for LXM in single form and in combination with other drugs. LXM is estimated by UV \& HPLC method. According to literature review, Eperisone is estimated by UV, HPLC, LC-EI-MS methods. There is no any method reported for Simultaneous estimation of LXM and EPE in a combination by UV and HPLC, but individually available for each drug and in combination with other drug. The present work describes the development of a simple, precise, accurate and reproducible spectrophotometric method for the simultaneous estimation of LXM and EPE in synthetic mixture. The developed method was validated in accordance with ICH Guidelines and successfully employed for the assay of LXM and EPE in synthetic mixture. 
Indo Global Journal of Pharmaceutical Sciences, 2015; 5(3): 225-232

\section{MATERIALS \& METHODS}

\section{Reagents and chemicals}

Analytically pure LXM and EPE were kindly provided by Cirex Pharmaceuticals Limited. Analytical grade methanol was purchased from Merck PVT LTD, India.

\section{Instrument and apparatus}

Shimadzu-1800 UV-Visible Spectrophotometer was used for spectral measurements with spectral band width $1 \mathrm{~nm}$, wavelength accuracy is $0.5 \mathrm{~nm}$ and $1 \mathrm{~cm}$ matched quartz cells. Software used was UV Probe (version 2.34). An Electronic analytical balance (Shimadzu) was used for weighing. Glassware used in each procedure were soaked overnight in a mixture of chromic acid and sulphuric acid rinsed thoroughly with double distilled water and dried in hot air oven.

\section{Method}

Absorbance ratio method uses the ratio of absorbance at two selected wavelengths, one which is an isoabsorptive point and other being the $\lambda$-max of one of the two components. From the overlay spectra of two drugs, it is evident that LXM and EPE show an isoabsorptive point at $291.5 \mathrm{~nm}$. The second wavelength used is $254.5 \mathrm{~nm}$, which is the $\lambda$-max of EPE. The concentration of two drugs in the mixture can be calculated using following equations. $\mathrm{CX}=[(\mathrm{QM}-\mathrm{QY}) /(\mathrm{QX}-\mathrm{QY})] \times \mathrm{A} 1 / \mathrm{aX} 1(1)$ $\mathrm{CY}=[(\mathrm{Qx}-\mathrm{Qm}) /(\mathrm{Qx}-\mathrm{Qy}) \times \mathrm{A} 1 / \mathrm{ay} 1$ (2) Where, $\mathrm{A} 1$ and A2 are absorbance of mixture at $291.5 \mathrm{~nm}$ and $254.5 \mathrm{~nm}$; $\mathrm{aX} 1$ and aY1 are absorptivities of LXM and EPE at 291.5 nm; aX2 and aY2 are absorptivities of LXM and EPE respectively at $254.5 \mathrm{~nm} ; \mathrm{QM}=\mathrm{A} 2 / \mathrm{A} 1, \mathrm{QX}=\mathrm{aX} 2 / \mathrm{aX} 1$ and $\mathrm{QY}=\mathrm{aY} 2 / \mathrm{aY} 1$.

\section{Preparation standard stock solutions}

Accurately weighed $10 \mathrm{mg}$ of LXM and EPE standard were transferred to separate $100 \mathrm{ml}$ volumetric flask and dissolved in $50 \mathrm{ml} 0.1 \mathrm{~N}$ methanolic $\mathrm{NaOH}$. The flasks were sonicated and volume was made up to the mark with the same solvent to give solutions containing $100 \mu \mathrm{g} / \mathrm{ml}$ LXM and $100 \mu \mathrm{g} / \mathrm{ml}$ EPE.

\section{Selection of Analytical Wavelength}

$2-14 \mu \mathrm{g} / \mathrm{ml}$ solutions of LXM and 2-16 $\mu \mathrm{g} / \mathrm{ml}$ solutions of EPE were prepared in methanol by appropriate dilution and spectrum was recorded between 200-500 $\mathrm{nm}$ and The overlain spectrums of LXM and EPE at different concentration were recorded. The isoabsorptive point was found to be $291.5 \mathrm{~nm}$ and maximum wavelength of EPE was found to be $254.5 \mathrm{~nm}$.

\section{METHOD VALIDATION}

The proposed method has been extensively validated in terms of specificity, linearity, accuracy, precision, limits of detection (LOD) and quantification (LOQ), robustness and reproducibility. The accuracy was expressed in terms of percent recovery of the known amount of the standard drugs added to the known amount of the pharmaceutical dosage forms. The precision (Coefficient of Variation C.V.) was expressed with respect to the repeatability, intra-day and inter-day variation in the expected drug concentrations. After validation, the developed methods have been applied to pharmaceutical dosage form.

\section{Specificity}

Commonly used excipients (starch, microcrystalline cellulose and magnesium stearate) were spiked into a pre weighed quantity of drugs.

\section{Linearity}

Appropriate volume of aliquot from LXM and EPE standard stock solution was transferred to volumetric flask of $10 \mathrm{ml}$ capacity. The volume was adjusted to the mark with methanol to give a solutions containing 2-16 $\mu \mathrm{g} / \mathrm{ml}$ of both LXM and EPE All Spectrum were recorded using above spectrophotometric condition. Absorbance at $291.5 \mathrm{~nm}$ and $254.5 \mathrm{~nm}$ were recorded for LXM and EPE, respectively $(n=6)$. Calibration curves were constructed by plotting average absorbance versus concentrations for both drugs. Straight line equations were obtained from these calibration curves.

\section{Accuracy}

Accuracy was assessed by determination of the recovery of the method by addition of standard drug to the prequantified placebo preparation at 3 different concentration levels 80, 100 and $120 \%$, taking into consideration percentage purity of added bulk drug samples. Each concentration was analyzed 3 times and average recoveries were measured.

\section{Precision}

The repeatability was evaluated by assaying 6 times of sample solution prepared for assay determination. The intraday and interday precision study of LXM and EPE was carried out by estimating different concentrations of LXM $(0.16,0.48,0.96 \mu \mathrm{g} / \mathrm{ml})$ and $\operatorname{EPE}(2,6,12 \mu \mathrm{g} / \mathrm{ml}), 3$ 


\section{Indo Global Journal of Pharmaceutical Sciences, 2015; 5(3): 225-232}

times on the same day and on 3 different days (first, second, third) and the results are reported in terms of C.V.

\section{Detection limit and Quantitation limit}

ICH guideline describes several approaches to determine the detection and quantitation limits. These include visual evaluation, signal-to-noise ratio and the use of standard deviation of the response and the slope of the calibration curve. In the present study, the LOD and LOQ were based on the third approach and were calculated according to the $3.3 \sigma / \mathrm{S}$ and $10 \sigma / \mathrm{S}$ criterions, respectively; where $\sigma$ is the standard deviation of $y$-intercepts of regression lines and $\mathrm{s}$ is the slope of the calibration curve.

\section{Robustness}

The sample solution was prepared and then analyzed with change in the typical analytical conditions like stability of analytical solution.

\section{Reproducibility}

The absorbance readings were measured at different laboratory for sample solution using another spectrophotometer by analyst and the values obtained were evaluated using t- test to verify their reproducibility. Determination of Lornoxicam and Eperisone in their synthetic mixture

\section{Sample preparation}

A powder quantity equivalent to $0.8 \mathrm{mg} \mathrm{LXM}$ and $10 \mathrm{mg}$ EPE was accurately weighed and transferred to volumetric flask of $100 \mathrm{ml}$ capacity. $60 \mathrm{ml}$ of $0.1 \mathrm{~N}$ methanolic $\mathrm{NaOH}$ was transferred to this volumetric flask and sonicated for $5 \mathrm{~min}$. The volume was made up to the mark with the same solvent. From this solution $0.2 \mathrm{ml}$ was transferred to volumetric flask of $10 \mathrm{ml}$ capacity. Volume was made up to the mark to give a solution containing $0.16 \mu \mathrm{g} / \mathrm{ml}$ of LXM and $2 \mu \mathrm{g} / \mathrm{ml}$ of EPE. The resulting solution was analyzed by proposed method. The quantitation was carried out by keeping these values to the straight line equation of calibration curve.

\section{RESULTS \& DISCUSSION}

Absorbance ratio method was developed for determination of LXM and EPE The proposed method has been extensively validated as per ICH guidelines. Considering above facts, wavelength $291.5 \mathrm{~nm}$ and 254.5 $\mathrm{nm}$ were selected for the estimation of LXM and EPE, respectively (figure 2). Linearity was assessed for LXM and EPE by plotting calibration curves of the absorbance versus the concentration over the concentration range 2$16 \mu \mathrm{g} / \mathrm{ml}$ for both drugs. The correlation coefficients (r2) for LXM and EPE were found to be 0.9986 and 0.997 , respectively (Table 2). The following equations for straight line were obtained for LXM and EPE. Linear equation for LXM, $y=0.0474 x+0.0465$ Linear equation for $\mathrm{EPE}, \mathrm{y}=0.0561 \mathrm{x}+0.0076$. The $\%$ recoveries were found to be in the range of $99.51 \pm 1.09$ for LXM and $99.57 \pm 1.44$ for EPE (Table 3 ). The precision of method was determined by repeatability, intraday and interday precision and was expressed as the C.V. (Table 1), which indicate good method precision. The Limit of detection for LXM and EPE was found to be $0.0052 \mu \mathrm{g} / \mathrm{ml}$ and $0.0042 \mu \mathrm{g} / \mathrm{ml}$ respectively. Limit of quantification for LXM and EPE was found to be $0.0158 \mu \mathrm{g} / \mathrm{ml}$ and 0.0127 $\mu \mathrm{g} / \mathrm{ml}$ at $291.5 \mathrm{~nm}$ and at $254.5 \mathrm{~nm}$ respectively (Table 1 ). The method was also found to be specific, as there was no interference observed when the drugs were estimated in presence of excipients and robust, as there was no significant change in absorbance up to 24 hours of preparation of solution in methanol. The proposed spectrophotometric method was successfully applied to LXM and EPE synthetic mixture and its combined dosage form. The results are shown in Table 6. 
Indo Global Journal of Pharmaceutical Sciences, 2015; 5(3): 225-232

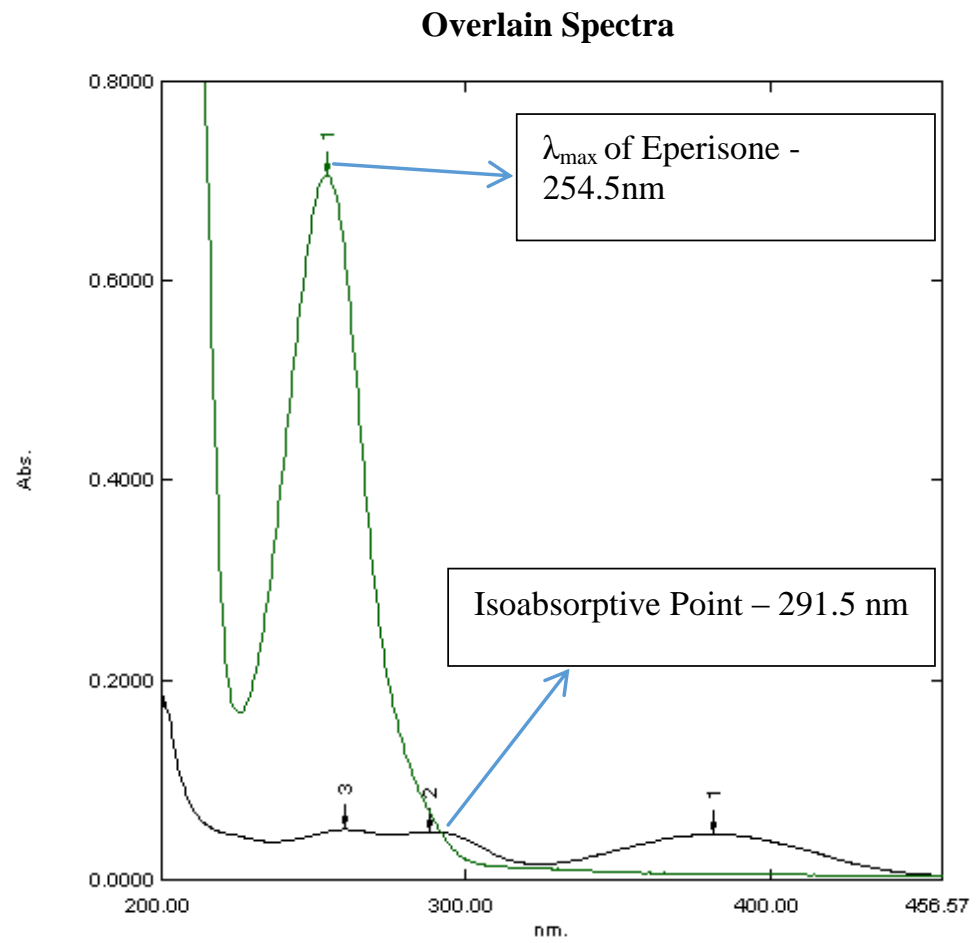

Calibration Curve of LXM

\begin{tabular}{|c|c|c|c|c|}
\hline Conc & $\begin{array}{c}\text { Abs at } \\
\mathbf{2 9 1 . 5} \\
\mathbf{n m}\end{array}$ & $\begin{array}{c}\text { Absorptivity- } \\
\mathbf{a}_{\mathbf{x} 1}\end{array}$ & $\begin{array}{c}\text { Abs at } \\
\mathbf{2 5 4 . 5} \\
\mathbf{n m}\end{array}$ & $\begin{array}{c}\text { Absorptivity } \\
\mathbf{a}_{\mathbf{x} 2}\end{array}$ \\
\hline 2 & 0.084 & 42 & 0.066 & 33 \\
\hline 4 & 0.162 & 40.5 & 0.148 & 37 \\
\hline 6 & 0.245 & 40.83 & 0.2 & 33.33 \\
\hline 8 & 0.352 & 44 & 0.289 & 36.125 \\
\hline 10 & 0.37 & 37 & 0.302 & 30.2 \\
\hline 12 & 0.452 & 37.67 & 0.368 & 30.67 \\
\hline & & Mean: 40.33 & & Mean: 33.38 \\
\hline
\end{tabular}

Calibration Curve of EPE

\begin{tabular}{|c|c|c|c|c|}
\hline Conc & $\begin{array}{c}\text { Abs at } \\
\mathbf{2 9 1 . 5} \\
\mathbf{n m}\end{array}$ & $\begin{array}{c}\text { Absorptivity- } \\
\mathbf{a}_{\mathbf{y} 1}\end{array}$ & $\begin{array}{c}\text { Abs at } \\
\mathbf{2 5 4 . 5} \\
\mathbf{n m}\end{array}$ & $\begin{array}{c}\text { Absorptivity- } \\
\mathbf{a}_{\mathbf{y} 2}\end{array}$ \\
\hline 2 & 0.015 & 7.5 & 0.122 & 61 \\
\hline 4 & 0.024 & 6 & 0.238 & 59.5 \\
\hline 6 & 0.031 & 5.17 & 0.336 & 56 \\
\hline 8 & 0.038 & 4.75 & 0.448 & 56 \\
\hline 12 & 0.047 & 3.92 & 0.617 & 51.42 \\
\hline 16 & 0.06 & 3.75 & 0.774 & 48.37 \\
\hline & & Mean: 5.18 & & Mean: 55.38 \\
\hline
\end{tabular}

\begin{tabular}{|c|c|c|}
\multicolumn{3}{|c}{ Mixture } \\
\hline $\mathbf{C x}+\mathbf{C y}$ & $\mathbf{A 1}$ & $\mathbf{A 2}$ \\
\hline $0.16+2$ & 0.0164 & 0.1057 \\
\hline $0.32+4$ & 0.0319 & 0.2234 \\
\hline $0.48+6$ & 0.0475 & 0.3149 \\
\hline $0.64+8$ & 0.0647 & 0.4524 \\
\hline $0.8+10$ & 0.0805 & 0.5419 \\
\hline $0.96+12$ & 0.0916 & 0.6725 \\
\hline
\end{tabular}


Indo Global Journal of Pharmaceutical Sciences, 2015; 5(3): 225-232

\begin{tabular}{|r|r|r|r|}
\hline \multicolumn{2}{|c|}{ Concentration $(\boldsymbol{\mu g} / \mathbf{m l})$} & $\begin{array}{c}\text { Absorbance of } \\
\text { LXM at 254 nm }\end{array}$ & $\begin{array}{c}\text { Absorbance of } \\
\text { EPE at 264.5 } \\
\text { nm }\end{array}$ \\
\hline \multirow{2}{*}{ LXM } & \multirow{2}{*}{ EPE } & & 0.122 \\
\hline 2 & 2 & 0.084 & 0.238 \\
\hline 6 & 4 & 0.163 & 0.336 \\
\hline 8 & 6 & 0.256 & 0.446 \\
\hline 10 & 8 & 0.353 & 0.617 \\
\hline 12 & 12 & 0.375 & 1.032 \\
\hline 14 & 16 & 0.453 & 1.221 \\
\hline 18 & 20 & 0.552 & \\
\hline 24 & 24 & 0.696 & 0.891 \\
\hline 28 & & 1.026 & \\
\hline
\end{tabular}

\section{Eperisone}

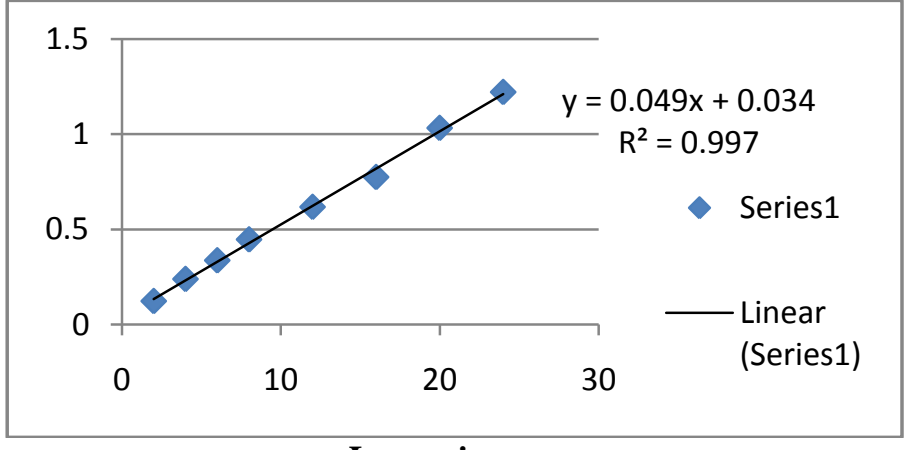

Lornoxicam

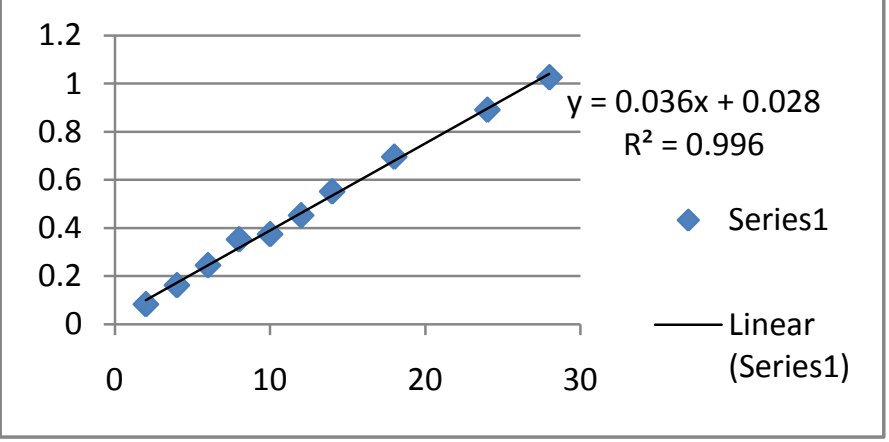

\begin{tabular}{|c|c|c|c|c|}
\hline Conc. & $\begin{array}{c}\text { Abs at } \\
\mathbf{2 9 1 . 5}\end{array}$ & $\begin{array}{c}\text { Conc. Of } \\
\text { LXM }\end{array}$ & $\begin{array}{c}\text { Abs at } \\
\mathbf{2 5 4 . 5}\end{array}$ & $\begin{array}{c}\text { Conc. Of } \\
\text { EPE }\end{array}$ \\
\hline \multirow{2}{*}{0.8 LXM } & 0.0805 & 0.76 & 0.5569 & 9.59 \\
\cline { 2 - 5 }+10.0 & 0.0806 & 0.766 & 0.5568 & 9.59 \\
\cline { 2 - 5 } EPE & 0.0804 & 0.761 & 0.5569 & 9.59 \\
\cline { 2 - 5 } & 0.0806 & 0.766 & 0.5568 & 9.59 \\
\cline { 2 - 5 } & 0.0805 & 0.76 & 0.5569 & 9.59 \\
\cline { 2 - 5 } & 0.0804 & 0.761 & 0.5567 & 9.59 \\
\hline Mean & & 0.762 & & 9.59 \\
\hline SD & & 0.0028 & & 0.0011 \\
\hline \% RSD & & 0.377 & & 0.012 \\
\hline
\end{tabular}

Reproducibility for the Intraday precision

\begin{tabular}{|c|c|c|c|c|c|c|c|c|c|}
\hline & \multirow[t]{2}{*}{$\begin{array}{l}\text { Wavelength } \\
\text { (nm) }\end{array}$} & \multicolumn{3}{|c|}{ Absorbance } & \multicolumn{3}{|c|}{$\begin{array}{c}\text { Concentration } \\
(\mu \mathrm{g} / \mathrm{ml})\end{array}$} & \multirow{2}{*}{$\begin{array}{l}\text { Mean } \\
\mu \mathrm{g} / \mathrm{ml} \\
\pm \text { S.D. }\end{array}$} & \multirow[t]{2}{*}{ RSD } \\
\hline & & $\mathbf{A}$ & B & $\mathbf{C}$ & $\mathbf{A}$ & B & $\mathrm{C}$ & & \\
\hline \multirow{2}{*}{$\begin{array}{c}2.16 \\
(0.16 \mathrm{~L}+ \\
2 \mathrm{E})\end{array}$} & 291.5 & 0.0164 & 0.0163 & 0.0165 & 0.16 & 0.16 & 0.16 & $0.16 \pm 0.0025$ & 1.53 \\
\hline & 254.5 & 0.1097 & 0.1095 & 0.1098 & 1.88 & 1.88 & 1.88 & $1.88 \pm 0.0011$ & 0.06 \\
\hline \multirow{2}{*}{$\begin{array}{c}6.48 \\
(0.48 \mathrm{~L}+ \\
6 \mathrm{E})\end{array}$} & 291.5 & 0.0478 & 0.0476 & 0.0479 & 0.45 & 0.45 & 0.45 & $0.45 \pm 0.0030$ & 0.67 \\
\hline & 254.5 & 0.3289 & 0.3285 & 0.3291 & 5.66 & 5.65 & 5.66 & $5.66 \pm 0.0035$ & 0.06 \\
\hline \multirow{2}{*}{$\begin{array}{c}12.96 \\
(0.16 \mathrm{~L}+ \\
12 \mathrm{E}) \\
\end{array}$} & 291.5 & 0.0976 & 0.0975 & 0.0977 & 0.92 & 0.92 & 0.92 & $0.92 \pm 0.0025$ & 0.27 \\
\hline & 254.5 & 0.6745 & 0.6744 & 0.6748 & 11.62 & 11.6 & 11.62 & $11.61 \pm 0.0083$ & 0.07 \\
\hline
\end{tabular}


Indo Global Journal of Pharmaceutical Sciences, 2015; 5(3): 225-232

Reproducibility study for the Interday Precision

\begin{tabular}{|c|c|c|c|c|c|c|c|c|c|}
\hline \multirow[t]{2}{*}{$\begin{array}{l}\text { Conc. } \\
(\mu \mathrm{g} / \mathrm{ml})\end{array}$} & \multirow[t]{2}{*}{$\begin{array}{l}\text { Wavelength } \\
\text { (nm) }\end{array}$} & \multicolumn{3}{|c|}{ Absorbance } & \multicolumn{3}{|c|}{$\begin{array}{c}\text { Concentration } \\
(\mu \mathrm{g} / \mathrm{ml})\end{array}$} & \multirow[t]{2}{*}{$\begin{array}{c}\text { Mean } \\
\mu \mathrm{g} / \mathrm{m} \pm \text { S.D. }\end{array}$} & \multirow[t]{2}{*}{ RSD } \\
\hline & & $\mathbf{A}$ & B & $\mathbf{C}$ & $\mathbf{A}$ & B & $\mathbf{C}$ & & \\
\hline \multirow{2}{*}{$\begin{array}{c}2.16 \\
(0.16 \mathrm{~L}+ \\
2 \mathrm{E})\end{array}$} & 291.5 & 0.0163 & 0.0163 & 0.0161 & 0.16 & 0.15 & 0.15 & $0.16 \pm 0.0026$ & 1.65 \\
\hline & 254.5 & 0.1093 & 0.1091 & 0.1088 & 1.87 & 1.87 & 1.87 & $1.874 \pm 0.001$ & 0.053 \\
\hline \multirow{2}{*}{$\begin{array}{c}6.48 \\
(0.48 \mathrm{~L}+ \\
6 \mathrm{E})\end{array}$} & 291.5 & 0.0476 & 0.0474 & 0.0471 & 0.45 & 0.44 & 0.44 & $0.447 \pm 0.006$ & 1.34 \\
\hline & 254.5 & 0.3285 & 0.3285 & 0.3279 & 5.65 & 5.65 & 5.65 & $5.655 \pm 0.0021$ & 0.036 \\
\hline \multirow{2}{*}{$\begin{array}{c}12.96 \\
(0.16 \mathrm{~L}+ \\
12 \mathrm{E}) \\
\end{array}$} & 291.5 & 0.0975 & 0.0973 & 0.0977 & 0.92 & 0.92 & 0.91 & $0.919 \pm 0.0045$ & 0.49 \\
\hline & 254.5 & 0.6744 & 0.6741 & 0.6748 & 11.62 & 11.61 & 11.61 & $11.616 \pm 0.0025$ & 0.021 \\
\hline
\end{tabular}

Specificity study for the synthetic mixture

\begin{tabular}{|c|c|c|c|c|c|c|}
\hline \multirow{2}{*}{$\begin{array}{c}\text { Conc. } \\
\boldsymbol{\mu g} / \mathbf{m l}\end{array}$} & $\begin{array}{c}\text { Max } \\
(\mathbf{n m} .)\end{array}$ & \multicolumn{2}{|c|}{$\begin{array}{c}\text { Before addition of } \\
\text { excipients }\end{array}$} & \multicolumn{2}{|c|}{$\begin{array}{c}\text { After addition of } \\
\text { excipients }\end{array}$} & $\begin{array}{c}\text { \% Inter- } \\
\text { ference }\end{array}$ \\
\cline { 2 - 6 } & & $\begin{array}{c}\text { Absorban } \\
\text { ce }\end{array}$ & $\begin{array}{c}\text { Conc. } \boldsymbol{\mu g} \\
/ \mathbf{m l}\end{array}$ & $\begin{array}{c}\text { Absorba } \\
\text { nce }\end{array}$ & $\begin{array}{c}\text { Conc. } \\
\boldsymbol{\mu g} / \mathbf{m l}\end{array}$ & \\
\hline $\begin{array}{c}4.32 \\
(0.32 \mathrm{~L} \\
+4 \mathrm{E})\end{array}$ & 291.5 & 0.0319 & 0.29 & 0.0318 & 0.29 & 0.68 \\
\cline { 2 - 6 } $\begin{array}{c}8.64 \\
(0.64 \mathrm{~L}+ \\
8 \mathrm{E})\end{array}$ & 254.5 & 0.2234 & 3.85 & 0.2231 & 3.85 & 0.10 \\
\cline { 2 - 6 } & 254.5 & 0.4524 & 7.78 & 0.4521 & 7.79 & 0.79 \\
\hline $\begin{array}{c}10.8 \\
(0.8 \mathrm{~L}\end{array}$ & 291.5 & 0.0805 & 0.76 & 0.0803 & 0.76 & 0.52 \\
\cline { 2 - 6 }$+10 \mathrm{E})$ & 254.5 & 0.5569 & 9.59 & 0.5565 & 9.59 & 0.052 \\
\hline
\end{tabular}

Accuracy

\begin{tabular}{|c|c|c|c|c|c|c|c|c|c|}
\hline $\begin{array}{c}\text { Mixt } \\
\text { ure } \\
\text { (LX } \\
\text { M: } \\
\text { EPH } \\
\text { ) }\end{array}$ & $\begin{array}{c}\text { Conc. } \\
(\mu \mathrm{g} / \\
\mathrm{ml})\end{array}$ & $\begin{array}{l}\text { Wavelen } \\
\text { gth }(\mathbf{n m})\end{array}$ & abs. & $\begin{array}{c}\text { Conc. } \\
\text { before } \\
\text { spiking } \\
(\mu \mathrm{g} / \mathrm{ml})\end{array}$ & $\begin{array}{c}\text { Refere } \\
\text { nce } \\
\text { standar } \\
\text { d } \\
\text { added } \\
(\mu \mathrm{g} / \mathrm{ml})\end{array}$ & abs. & $\begin{array}{c}\text { Conc. } \\
\text { after } \\
\text { spiking } \\
(\mu \mathrm{g} / \mathrm{ml})\end{array}$ & $\begin{array}{c}\text { Actual } \\
\text { conc. }\end{array}$ & $\begin{array}{c}\% \\
\text { Recovery }\end{array}$ \\
\hline \multirow[t]{2}{*}{1} & \multirow{6}{*}{ 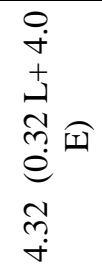 } & 291.5 & 0.0319 & 0.29 & $80 \%$ & 0.0583 & 0.54 & 0.25 & 98.43 \\
\hline & & 254.5 & 0.2234 & 3.85 & $80 \%$ & 0.4056 & 6.99 & 3.14 & 98.06 \\
\hline \multirow[t]{2}{*}{2} & & 291.5 & 0.0318 & 0.29 & $100 \%$ & 0.0641 & 0.61 & 0.32 & 100.62 \\
\hline & & 254.5 & 0.2232 & 3.85 & $100 \%$ & 0.4576 & 7.89 & 4.04 & 100.95 \\
\hline \multirow[t]{2}{*}{3} & & 291.5 & 0.0319 & 0.29 & $120 \%$ & 0.0701 & 0.68 & 0.38 & 99.48 \\
\hline & & 254.5 & 0.2234 & 3.85 & $120 \%$ & 0.0907 & 8.64 & 4.78 & 99.71 \\
\hline \multicolumn{2}{|r|}{ SD } & \multicolumn{2}{|c|}{$\begin{array}{c}\text { LXM } \\
99.51 \pm 1.09\end{array}$} & $\begin{array}{c}\mathrm{EPE} \\
.57 \pm 1.44\end{array}$ & \multicolumn{2}{|c|}{ \%RSD } & \multicolumn{2}{|l|}{$\begin{array}{c}\text { LXM } \\
1.09\end{array}$} & $\begin{array}{l}\text { EPE } \\
1.45\end{array}$ \\
\hline
\end{tabular}


Indo Global Journal of Pharmaceutical Sciences, 2015; 5(3): 225-232

LOD \& LOQ

\begin{tabular}{|c|c|c|c|c|c|c|}
\hline S. No. & $\begin{array}{c}\text { ABS of } \\
\text { the } \\
\text { blank at } \\
291.5 \\
\text { nm }\end{array}$ & $\begin{array}{c}\text { ABS of } \\
\text { the } \\
\text { blank at } \\
254.5 n \\
\text { m }\end{array}$ & $\begin{array}{c}\text { Slope of } \\
\text { calibratio } \\
\text { n curve at } \\
291.5 \mathrm{~nm}\end{array}$ & $\begin{array}{c}\text { Slope of } \\
\text { calibratio } \\
\text { n curve at } \\
254.5 \mathrm{~nm}\end{array}$ & $\begin{array}{l}\text { L.O.D. } \\
\mu \mathrm{g} / \mathrm{ml}\end{array}$ & $\begin{array}{l}\text { L.O.Q. } \\
\mu \mathrm{g} / \mathrm{ml}\end{array}$ \\
\hline 1 & 0.0164 & 0.1097 & \multirow[t]{6}{*}{0.0474} & \multirow[t]{6}{*}{0.0963} & \multirow{4}{*}{$\begin{array}{c}\text { LXM } \\
0.0052\end{array}$} & \multirow{4}{*}{$\begin{array}{l}\text { LXM } \\
0.015\end{array}$} \\
\hline 2 & 0.0163 & 0.1095 & & & & \\
\hline 3 & 0.0164 & 0.1097 & & & & \\
\hline 4 & 0.0163 & 0.1095 & & & & \\
\hline 5 & 0.0164 & 0.1097 & & & \multirow{4}{*}{$\begin{array}{c}\text { EPE } \\
0.0042\end{array}$} & \multirow{4}{*}{$\begin{array}{c}\text { EPE } \\
0.013\end{array}$} \\
\hline 6 & 0.0165 & 0.1098 & & & & \\
\hline Mean & 0.0163 & 0.1096 & & & & \\
\hline S.D. & $\begin{array}{c}7.53 \times 10^{-} \\
\end{array}$ & $\underset{4}{1.22 \times 10^{-}}$ & & & & \\
\hline
\end{tabular}

Robustness Study for the synthetic mixture at $291.5 \& 254.5 \mathrm{~nm}$

\begin{tabular}{|c|c|c|c|c|c|c|c|c|c|}
\hline \multirow{2}{*}{$\begin{array}{c}\text { Conc. } \\
(\mu \mathrm{g} / \mathrm{ml} \\
)\end{array}$} & \multirow{2}{*}{$\begin{array}{c}\text { Wavel } \\
\text { ength } \\
(\mathbf{n m})\end{array}$} & \multicolumn{3}{|c|}{ Absorbance } & \multicolumn{3}{|c|}{$\begin{array}{c}\text { Concentration } \\
(\mu \mathrm{g} / \mathrm{ml})\end{array}$} & \multirow{2}{*}{$\begin{array}{l}\text { Mean } \\
\mu \mathrm{g} / \mathrm{ml} \\
\pm \text { S.D. }\end{array}$} & \multirow[t]{2}{*}{ RSD } \\
\hline & & $\mathbf{A}$ & B & $\mathrm{C}$ & $\mathbf{A}$ & B & C & & \\
\hline \multirow{6}{*}{$\begin{array}{c}10.8 \\
(0.8 \mathrm{~L}+ \\
10 \mathrm{E})\end{array}$} & 290.5 & 0.0809 & 0.0808 & 0.0809 & 0.773 & 0.771 & 0.773 & $\begin{array}{l}0.772 \pm \\
0.0011\end{array}$ & 0.15 \\
\hline & 253.5 & 0.5571 & 0.5569 & 0.5571 & 9.592 & 9.59 & 9.592 & $\begin{array}{l}9.591 \pm \\
0.0011\end{array}$ & 0.012 \\
\hline & 291.5 & 0.0805 & 0.0805 & 0.0804 & 0.763 & 0.763 & 0.761 & $\begin{array}{l}0.762 \pm \\
0.0012\end{array}$ & 0.15 \\
\hline & 254.5 & 0.5569 & 0.5569 & 0.5568 & 9.595 & 9.595 & 9.593 & $\begin{array}{l}.594 \pm \\
0.0011 \\
\end{array}$ & 0.012 \\
\hline & 292.5 & 0.0801 & 0.0802 & 0.0801 & 0.753 & 0.756 & 0.753 & $\begin{array}{l}0.754 \pm \\
0.0017\end{array}$ & 0.23 \\
\hline & 255.5 & 0.5564 & 0.5566 & 0.5565 & 9.592 & 9.594 & 9.592 & $\begin{array}{l}9.592 \pm \\
0.0012\end{array}$ & 0.012 \\
\hline
\end{tabular}

(Vadodara, India) for providing the necessary facilities for

\section{CONCLUSION}

The proposed first order derivative method provide simple, specific, precise, accurate and reproducible quantitative analysis for simultaneous determination of LXM and EPE in synthetic mixture. The method was validated as per ICH guidelines in terms of specificity, linearity, accuracy, precision, limits of detection (LOD) and quantification (LOQ), robustness and reproducibility. The proposed method can be used for routine analysis and quality control assay of LXM and EPE in combined dosage form.

\section{ACKNOWLEDGMENT}

Authors are thankful to Cirex Pharmaceuticals Limited, Hydrabad for providing gratis sample. The authors also thankful to Parul Institute of Pharmacy and Research research work.

\section{REFERENCES}

1. Beckett A.H, Stenlake J.B, UV-visible Spectrophotometry: Practical Pharmaceutical Chemistry, $4^{\text {th }}$ edn, Part-II, C.B.S. Publishers, Delhi, 285-97(2001).

2. Sharma B.K, Instrumental Methods of Chemical Analysis, Goel Publication House, Meerut, pp 133-161.

3. Sharma Y.R, Ultraviolet and visible spectroscopy in Elementary Organic Spectroscopy, $1^{\text {st }}$ edn, S. Chand \& Company Ltd., New Delhi, 9-60 (2004).

4. Meyer Veronica R, Practical High Performance Liquid Chromatography, $2^{\text {nd }}$ edn, John Wiley and Sons, London, 26258, (1993)

5. Kazakevich Y, Lobrutto R, HPLC for Pharmaceutical Scientists, $1^{\text {st }}$ edn, John Wiley \& Sons Inc., HobokenNew jersey, (2007).

6. Snyder L.R, Kirkland J.L, Glajch J.L, Practical HPLC Method Development, New York, Wiley, (1997). 
Indo Global Journal of Pharmaceutical Sciences, 2015; 5(3): 225-232

7. Singh Y.k, Fundamental of Research Methodology and Statistics, New Age International Publishers, 238-240 (2000)

8. Boltan S. Bonc, Pharmaceutical statistics Practical and Clinical Applications, $4^{\text {th }}$ Edn, Vol. marcel Dekker, 215

9. Chan C.C, Analytical Method Validation and Instrument Performance Verification, 24-26.

10. International conference on harmonization of technical requirements for registration of pharmaceuticals for human use. Validation of analytical procedures: Text and Methodology ICH Q2 (R1), (2005).

11. Prajapati $P$, Vaghela V, Rawtani $D$, Patel H, Kubavat J, Baraiya $\mathrm{D}$, "Azeotropic mixture used for development and validationof Lornoxicam in bulk and its tablet dosage formby spectrophotometric method" Journal of Pharmaceutical Analysis, 2(4),306-309(2012)

12. Sahoo S.K, Giri R.K, Patil S.V, Behera A.R and Mohapatra R, "Development of ultraviolet spectrophotometric method for analysis of Lornoxicam in solid dosage forms" Tropical Journal of Pharmaceutical Research,11(2),269-273 (2012)

13. Patel A, Patel N, Patel M, Lodha A, Chaudhuri J, Jadia P, Joshi T, Dalal J, "Development and validation of anlytical methods for the simultaneous estimation of Lornoxicam and Paracetamol from their pharmaceutical dosage form", IOSR Journal of Pharmacy, 2(3), 364-366 (2012)

14. Bendale A.R, Makwana J.J, Narkhede S.P, Jadhav A.J, Vidyasagar G," Analytical method development and validation protocol forLornoxicam in tablet dosage form", Journal of Chemicghal and Pharmaceutical Research,3(2),258-263 (2011)

15. Warkar C.B and Rele R.V, "Estimation of Lornoxicam and Diacerein in dosage form by simultaneous equation and Qanalysis method using UV spectroscopic technique", International Journal of Pharma and Bio Sciences, 2(2),124129(2011)

16. Mokale S.N, Nirmal S.S, Lahoti S.R, Sangshetti J.N, "Simultaneous estimation of Paracetamol and Lornoxicam by RP-HPLC method from combined dosage forms", Der Pharmacia Sinica, 2 (5),138-144 (2011)

17. Shah D.A, Patel N.J, Baldania S.L, Chhalotiya U.K , Bhatt K.K, "Stability Indicating LC-Method for Estimation of Paracetamol and Lornoxicam in Combined Dosage Form", Scientia Pharmaceutica,79,113-122 (2011)
18. Jain D, Dubey N, Sharma Y, "Development and validation of a stability-indicating HPTLC method and HPLC method for simultaneous estimation of Lornoxicam and Paracetamol in combined tablet dosage forms", Int J Pharm Biomed Sci, 2(2)55-60,( 2011)

19. Kuchekar B.S, Sahoo M, Syal P, Ingale S, Ingale K, Sindhe S, Sali M, Choudhari V.P, "Development and Validation of a RPHPLC-PDA method for Simultaneous Determination of Lornoxicam and Thiocolchicoside in Pharmaceutical dosage form and its Application for Dissolution study", International Journal of Research in Pharmaceutical Sciences, 2(1),1-7(2011)

20. Singh B, Saini G, Sharma D.N, Roy S. D and Gautam N, "Estimation of Lornoxicam in tablet dosage form by UV spectrophotometric method", International Journal of Pharmaceutical Sciences and Research, 2(1),102-106(2011)

21. Attimarad M, "Rapid RP-HPLC method for quantitative determination of Lornoxicam in tablets", Journal of Basic and Clinical Pharmacy, 1(2)115-118(2010)

22. Jain $\mathrm{N}$, Jain $\mathrm{R}$, Sahu V, Sharma $H$, Jain $S$, Jain $D$, "Spectrophotometric quantitative estimation of Lornoxicam and Paracetamol in Bulk Drugs and Dosage Form", Der Pharma Chemica, 2(6)165-170(2010)

23. Lakshmi S, Lakshmi K.S And Tintu T, "Simultaneous spectrophotometric estimation of Paracetamol and Lornoxicam in tablet dosage form", International Journal of Pharmacy and Pharmaceutical Sciences, 2(4),166-168 (2010)

24. Bhavsar K. C, Gaikwad P. D, Bankar V. H and Pawar S. P, "Development and validation of UV spectrophotometric method for simultaneous estimation of Paracetamol and Lornoxicam in bulk and tablet dosage form", International Journal Of Pharmacy \& Technology,2(2), 429-439(2010)

25. Patel U.J Patel P.U, Patel S.K, "Spectrophotometric method fpr simultaneous estimation of Eperisone Hydrochloride and Diclofenac Sodium in synthetic mixture", International Research journal of pharmacy, 3(1),203-206 (2012)

26. Ding L, Wei $X$, Zhang $S$, Sheng J, Zhang Y, "Rapid and sensitive liquid chromatography-electroscopy ionization-mass spectrometry method for the determination of Eperisone in human plasma: Method and clinical applications", Journol of chromatographic Science, 42, 254-258,( 2004)

Indo Global Journal of Pharmaceutical Sciences( ISSN 22491023 ; CODEN- IGJPAI; NLM ID: 101610675) indexed and abstracted in EMBASE(Elsevier), SCIRUS(Elsevier),CABI, CAB Abstracts, Chemical Abstract Services(CAS), American Chemical Society(ACS), Index Copernicus, EBSCO, DOAJ, Google Scholar and many more. For further details, visit 